\title{
Smart Metering Information Management
}

\section{DOI 10.1007/s11576-011-0285-z}

\section{Die Autoren}

Dipl.-Wirtsch.-Ing.

Ullrich C. C. Jagstaidt ( $\varangle)$

Janis Kossahl, M. Sc.

Prof. Dr. Lutz M. Kolbe

Professur für

Informationsmanagement

Georg-August-Universität Göttingen

Platz der Göttinger Sieben 5

37073 Göttingen

Deutschland

ullrich.jagstaidt@wiwi.uni-

goettingen.de

janis.kossahl@wiwi.uni-

goettingen.de

lutz.kolbe@wiwi.uni-goettingen.de

Eingegangen: 2010-11-22

Angenommen: 2011-06-30

Angenommen nach zwei Überarbei-

tungen durch Prof. Dr. Sinz.

Online publiziert: 2011-08-20

This article is also available in English via http://www.springerlink.com and http://www.bise-journal.org: Jagstaidt UCC, Kossahl J, Kolbe LM (2011) Smart Metering Information Management. Bus Inf Syst Eng. doi: 10.1007/s12599-011-0173-5.

(C) The Author(s) 2011. Dieser Artikel ist auf Springerlink.com mit Open Access verfügbar

\section{Informationsmanagement in der Energiewirtschaft}

Die Energiewirtschaft ist derzeitig in einem tiefgreifenden Wandel. Insbesondere gilt dies für die Energieerzeugung durch die Zielsetzung der Bundesregierung, bis zum Jahr 2050 den Anteil der Stromerzeugung aus erneuerbaren Energien auf $80 \%$ zu steigern (BMU und BMWi 2010). Aber auch bei der Stromübertragung und dem Absatz an die Endverbraucher sind die beteiligten Akteure seit Beginn der Liberalisierung des Strommarktes mit erheblichen Veränderungen konfrontiert.

Mit diesen Entwicklungen werden zusätzliche Herausforderungen an das In- formationsmanagement in der Energiewirtschaft gestellt. Zur Differenzierung wird in Anlehnung an den Business Engineering Ansatz (Österle und Winter 2003) das 3-Ebenenmodell eingeführt. Hiermit können die Akteure in ihrer unterschiedlichen Wertschöpfungsrolle, die Informationen nach Ausprägungen und Prozess sowie die Infrastruktur nach wesentlichen Systemelementen eingeordnet und im Zusammenhang berücksichtigt werden (siehe Abb. 1).

Die Informationsebene dient dabei als eigenständiges und verbindendes Element, sowohl zum vertikalen Datenaustausch zwischen der technischen Infrastruktur der Netze und den Akteuren der Stromwirtschaft als auch zur automatisierten Steuerung von Informationsflüssen innerhalb der Akteurs- oder der Infrastrukturebene.

Auf der Infrastrukturebene kann der derzeitige, durch das Erneuerbare Energien Gesetz (EEG) geförderte, Ausbau der regenerativen Energien eingeordnet werden. Da der Großteil der Stromerzeugung aus regenerativen Energien deutlich volatiler als die Stromgewinnung aus fossilen Energieträgern ist, steigt die Komplexität der Netzsteuerung zur Ausbalancierung von Einspeisung und Abnahme deutlich.

Mit der seit 1998 begonnenen Liberalisierung der Strombranche ist die Anzahl der Akteure gestiegen. Während vormals die vier großen Energieversorgungsunternehmen als Monopolanbieter die gesamte Wertschöpfungskette von Erzeugung, Transport und Vertrieb abgedeckt haben, sind die Unternehmen seit dem Inkrafttreten des Energiewirtschaftsgesetzes (EnWG 2005) dazu verpflichtet, ihre Sparten organisatorisch und bilanzrechtlich voneinander $\mathrm{zu}$ trennen (das sogenannte Unbundling).

Während es auf den Akteurs- und Infrastrukturebenen in den letzten Jahren viele Neuerungen gab, sind die Standards, Prozesse und Geschäftsmodelle der Informationsebene bis heute noch in der Findungsphase.

\section{Smart Metering und Smart Grid}

Die Vision für das zukünftige Energieversorgungssystem besteht darin, durch die Einführung neuer, „intelligenter“ Technologien die wichtiger werdende Netz-
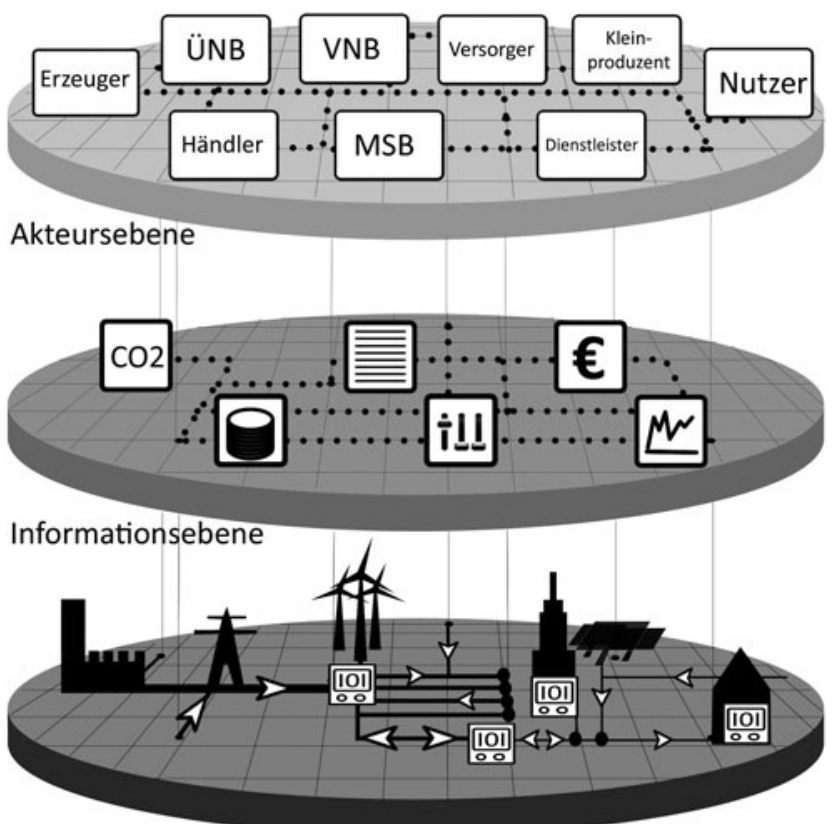

Infrastrukturebene

Abb. 1 Ebenenmodell der Energiewirtschaft (BMWi 2011b) 
Abb. 2 Die Verbindung von Smart Metering und Smart Grid

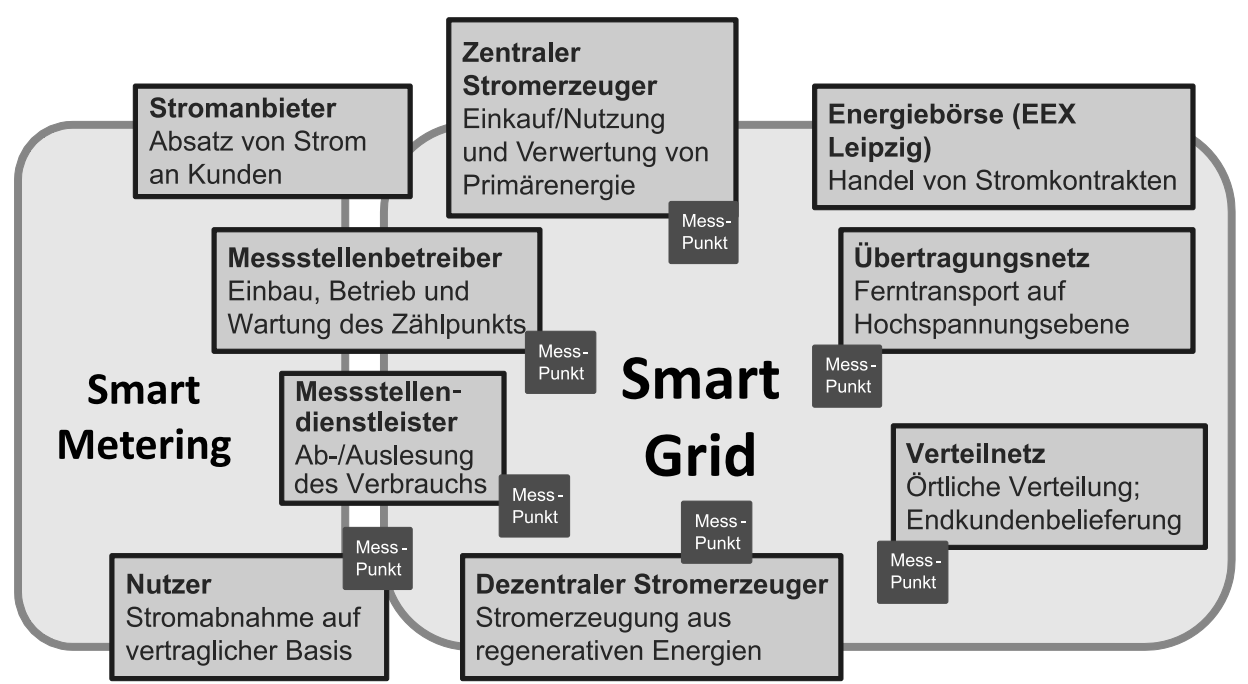

steuerung in den Verteilnetzen zu ermöglichen. Zusätzlich sollen Kunden durch neue Tarifmodelle Anreize zum erzeugungsorientierten Verbrauch und Anbieter durch den Vertrieb von zusätzlichen Funktionalitäten neue Wachstumschancen erhalten.

Diese Funktionalitäten sollen in einem Smart Grid verfügbar werden. Der Begriff „umfasst die Vernetzung und Steuerung von intelligenten Erzeugern, Speichern, Verbrauchern und Netzbetriebsmitteln in Energieübertragungs- und Verteilnetzen mit Hilfe von Informationsund Kommunikationstechnik (IKT)“ (VDE/ITG 2010).

Grundlegende Voraussetzung für die Erreichung eines „intelligenten“ Energieversorgungssystems ist der Einsatz von digitalen Stromzählern (sogenannte Smart Meter) an Stelle des analogen Ferraris-Zählers. Der Smart Meter ist sowohl der digitale Messpunkt für den Energieverbrauch im Haushalt, als auch ein zu etablierendes Steuerungselement im lokalen Verteilnetz. Smart Meter können sinnvollerweise mit einem Kommunikationsmodul für die Ermöglichung des bidirektionalen Informationsflusses ausgestattet werden. Der Datentransfer kann durch verschiedene Übertragungstechniken realisiert werden (z. B. Power Line Communication, Mobilfunk, Breitband).

Durch den Einsatz einer Vielzahl von verteilten Messpunkten wird es erst möglich, die zur Steuerung benötigten Informationen über den Zustand des physikalischen Stromnetzes zu erhalten (Watson et al. 2010).

Das Smart Metering umfasst den Informationsverarbeitungsprozess, in dem
Verbrauchs- und Nutzungsdaten gesammelt, evaluiert und aggregiert, Prüfungen und Diagnosen von Stromspannung, Lastabnahme und Betriebszustand in Echtzeit durchführt sowie Steuerungssignale weitergeleitet werden. Die Daten können hierbei in unterschiedlicher Granularität und in verschiedenen zeitlichen Intervallen gemessen und verarbeitet werden.

Während der Begriff Smart Grid auch die Steuerung der Stromeinspeisung beinhaltet, beispielsweise die Steuerung verschiedener regenerativer Energiequellen als virtuelle Kraftwerke zur Grundlastversorgung, stehen beim Smart Metering die durch die Einführung von digitalen Stromzählern entstehenden Möglichkeiten z. B. für Tarifierung und Preisgestaltung im Vordergrund (siehe Abb. 2).

Nach der Sichtweise der European Smart Metering Alliance (ESMA) steht der Kunde im Mittelpunkt: „Smart Metering is designed to provide utility customers information on a real time basis about their domestic energy consumption. This information includes data on how much gas and electricity they are consuming, how much it is costing them and what impact their consumption is having on greenhouse gas emissions" (ESMA 2010).

Ein durchgängiger meter-to-cashProzess kann dann verwirklicht werden, wenn die beim Smart Metering entstehenden Informationen in einer integrierten Systemlandschaft automatisiert erhoben, transferiert, ausgewertet und an die Anwendungen zur weiteren Nutzung übertragen werden.

\section{Stand der Entwicklung des Smart Metering}

Mit den EU-Richtlinien zur Energie- und Klimapolitik (Europäische Union 2006) und der Umsetzungsverordnung zur Einführung neuer Anreize zur Steuerung und Energieeinsparung (EnWG $\$ 21$, $\$ 40$ ) wurden die gesetzlichen Grundlagen für den Einsatz intelligenter Energieverbrauchsmessgeräte sowie zur Liberalisierung des Messstellenbetriebes in Deutschland geschaffen.

Unter der Maßgabe der von der EU-Kommission beschlossenen 20-2020 Regelung (20\% höhere Energieeffizienz, $20 \%$ Anteil von erneuerbaren Energien am Gesamtenergieverbrauch und $20 \%$ weniger Treibhausgasemissionen) soll die Umstellung von den herkömmlichen Ferraris-Stromzählern hin zu intelligenten Messpunkten bis zum Jahr 2020 in $80 \%$ der Haushalte abgeschlossen sein (EU-Kommission 2007).

Aktuell sind für das Smart Metering insbesondere die Paragraphen $\$ 21 \mathrm{~b}$ und $\$ 40$ des EnWG relevant. Demnach müssen seit Januar 2010 bei allen Neubauten und grundlegenden Sanierungen digitale Stromzähler eingebaut werden. Durch $\$ 40$ sind Stromanbieter seit dem Januar 2011 gesetzlich dazu verpflichtet, einen Tarif anzubieten, der Anreize zur Energieeinsparung oder Steuerung des Energieverbrauchs setzt. Darunter sind lastvariable oder tageszeitabhängige Tarife zu verstehen.

Neben diesen regulatorischen Bemühungen wird vom Bundesministerium für Wirtschaft und Technologie (BMWi) aber auch deutlich gemacht, dass es im Zählerwesen „Vorrang für mehr Wettbewerb“ (BMWi 2011a) geben soll. 
Tab. 1 Stand der Smart Meter Einführung in Europa (ERGEG 2007)

\begin{tabular}{|c|c|c|c|c|}
\hline $\begin{array}{l}\text { Einführung von Smart } \\
\text { Metern (SM) nach } \\
\text { europäischen Ländern }\end{array}$ & $\begin{array}{l}\text { Liberalisierter Markt für } \\
\text { SM und Messstellen- } \\
\text { Betreiber (MSB) }\end{array}$ & $\begin{array}{l}\text { Versorgungsgrad mit SM } \\
\text { in Haushalte }(\mathrm{HH}) \text { (in \% } \\
\text { und Jahr) }\end{array}$ & $\begin{array}{l}\text { Kommentare zur } \\
\text { Entwicklung }\end{array}$ & $\begin{array}{l}\text { Verantwortung für den } \\
\text { Smart Meter Rollout }\end{array}$ \\
\hline Niederlande & Ja, seit 2000 & $3 \%(2008)$ & $\begin{array}{l}\text { Neuregelung der } \\
\text { Verantwortung in } 2008\end{array}$ & $\begin{array}{l}\text { Nun regionaler } \\
\text { Verteilnetzbetreiber }\end{array}$ \\
\hline Großbritannien & $\begin{array}{l}\text { Ja, in einzelnen Schritten } \\
\text { von } 2000-2005\end{array}$ & $>0,5 \%(2007)$ & $\begin{array}{l}\text { Nun Konsolidierung der } \\
\text { Verfahrensweisen bis } \\
\text { Ende } 2010\end{array}$ & $\begin{array}{l}\text { MSB für SM, } \\
\text { Netzbetreiber für } \\
\text { Dienstleistung }\end{array}$ \\
\hline Italien & $\begin{array}{l}\text { Nein, Ausbau mit } \\
\text { staatlicher Billigung } \\
\text { 2001-2005 }\end{array}$ & $86 \%$ (2008) & $\begin{array}{l}\text { Eigeninitiative des } \\
\text { Versorgers ENEL }\end{array}$ & Verteilnetzbetreiber \\
\hline Schweden & $\begin{array}{l}\text { Nein, nur Vorgaben zur } \\
\text { Abrechnung }\end{array}$ & $59 \%(2009)$ & $\begin{array}{l}\text { Hoher Ausbaugrad mit } \\
\text { innovativen Zählern und } \\
\text { bereits } 97 \% \\
\text { Unternehmen }\end{array}$ & $\begin{array}{l}\text { Regionaler } \\
\text { Verteilnetzbetreiber }\end{array}$ \\
\hline Deutschland & Ja, seit 2008 & Pilotprojekte & $\begin{array}{l}\text { Keine Vorgaben für den } \\
\text { Ausbau }\end{array}$ & Offen \\
\hline
\end{tabular}

Auch die Bundesnetzagentur empfiehlt das „Absehen von der staatlichen Vorgabe eines flächendeckenden Rollouts und einer vorzeitigen Festlegung auf eine nationale Ausbauquote“ (BMWi 2011a).

Da bislang keiner der Akteure der Energiewirtschaft eigenverantwortlich die Initiative ergriffen hat, gibt es in Deutschland zwar derzeitig eine Vielzahl von Pilotprojekten, aber keine Bestrebungen zur flächendeckenden Einführung. Die weitgehend regulierten Verteilnetzbetreiber warten primär auf Veränderungen bei der Anreizregulierung und ein klares rechtliches Mandat, während die Stromanbieter die Refinanzierbarkeit der erheblichen Investition bezweifeln. Zusätzlich wird von den Beteiligten das Fehlen von Geschäftsmodellen, Organisationsstrukturen und Standards zur Gewährleistung der Interoperabilität kritisch gesehen.

Die überwiegend mit öffentlichen Mitteln geförderten Smart-MeteringPilotprojekte in Deutschland dienen vorwiegend dazu, sich bereits jetzt aktiv auf die zukünftigen Anforderungen vorzubereiten und die technologischen Möglichkeiten in ihrer gesamten Bandbreite $\mathrm{zu}$ testen (Müller-Elschner 2010).

Untersuchungen zeigen den unterschiedlichen Stand und das unterschiedliche Tempo der weltweiten Bemühungen, Smart Meter flächendeckend einzuführen (siehe Tab. 1). Es wird dabei deutlich, dass den nationalen und staatlichen Richtlinien eine wichtige Rolle bei der Gestaltung der Entwicklungen zukommt.

In den USA und in Australien werden bereits jetzt Verfahren wie das Demand
Response (DR) erprobt, mit denen Anreize zur Lastverschiebung in günstigere Zeit- und Tarifmodelle geprobt werden. Analysen haben gezeigt, dass sich bereits bei wenigen Nutzern eine Kosteneinsparung von ca. $3 \%$ erzielen lässt. Der Gesamtenergiebedarf insgesamt sinkt zwar nicht signifikant, jedoch kann über den Tag eine bessere Gesamtbilanzausnutzung erzielt werden (Fox-Penner 2010). Andere Untersuchungen zielen auf die Bereiche der gesteuerten vertikalen Interaktion, durch die Netzwerkosten reduziert und Dienstleistungen entlang der Energiewertschöpfung verbessert werden können (VDE/ITG 2010).

\section{Bedeutung des Informationsmanagements für das Smart Metering}

Die vielfältigen Veränderungen bedeuten einen Paradigmenwechsel in der Energiewirtschaft. Aus der unidirektionalen Energieverteilung vom Erzeuger zum Nutzer mit stetig abnehmenden Spannungen wird durch die dezentrale Einspeisung erneuerbarer Energien eine n:m-Beziehung: aus Nutzern werden auch Produzenten („Prosumer“), aus Verteilungsmodellen werden lastabhängige Nachfrage-Modelle, aus stabilen werden dynamische Prozesse. Diese Veränderungen haben nicht nur erheblichen Einfluss auf die beteiligten Akteure, sondern auch auf das Daten- und Informationsmanagement. Die zentralen Herausforderungen für das Informationsmanagement beim Smart Metering werden in Abb. 3 für die drei Ebenen des Informationsmanagements nach Heinrich und Lehner (2005) dargestellt.

In den nächsten Jahren werden die strategischen Aufgaben für das Informationsmanagement darin bestehen, durch die unumgängliche Einbindung von Informations- und Kommunikationstechnologien in allen Bereiche der Wertschöpfung der Energiewirtschaft, nachhaltige Geschäftsmodelle zu entwickeln und zu bewerten.

Darüber hinaus wird durch die Einführung des Smart Metering nicht der quantitative Stromabsatz steigen, vielmehr werden die Konsumenten voraussichtlich verbrauchssensibler werden. Stromanbieter müssen sich darauf einstellen und neue, IT-getriebene Geschäftsmodelle und weitergehende Konzepte zur Kundenbindung entwickeln, da der reine Stromverkauf zusehends zur Commodity wird. Innovative Anbieter und Dienstleister werden auf den Markt drängen, die nicht aus der Tradition eines Energieversorgungsunternehmens kommen.

Es werden Analogien zur Entwicklung der Telekommunikationsbranche deutlich. Die signifikantesten Merkmale sind dabei der forcierte Wandel zu mehr Wettbewerb, steigende Wahlmöglichkeiten der Nutzer, die stärkere Einbindung der IT in die Unternehmenslandschaft insbesondere durch die extreme Zunahme des unternehmensinternen und übergreifenden Datenaustausches.

Für die effektive und zielführende Interaktion der Akteure untereinander zur Ermöglichung des Smart Meterings und auch darüber hinaus zur Ausbalancie- 
Abb. 3 Herausforderungen für das Informationsmanagement beim Smart Metering

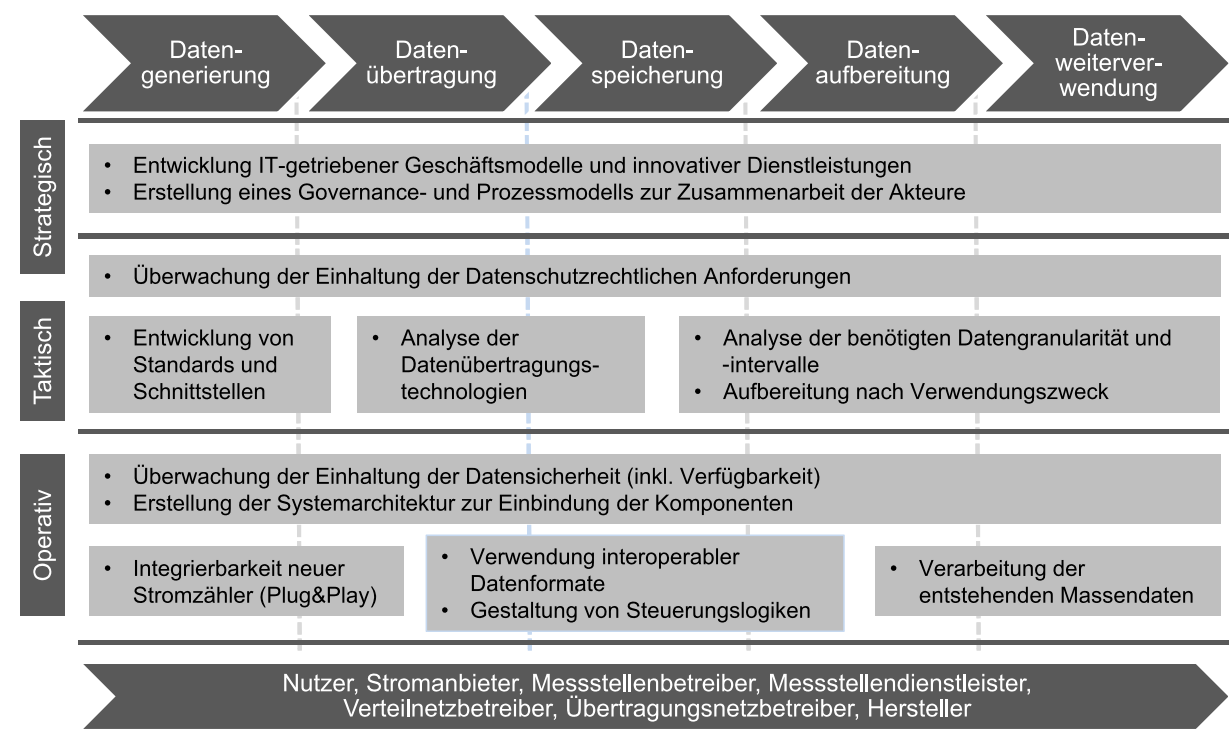

rung von Energieeinspeisung und Lastentnahme ist es wichtig, ein möglichst konsensuales Governance-Rahmenwerk zu entwickeln. Dieses sollte durch Leitlinien und Prozessdefinitionen aufzeigen, welche Marktrolle welche Verantwortlichkeit zur Generierung, Weiterleitung, Speicherung und Verwendung der gewonnenen Daten erhält.

Beim Smart Metering entstehen ebenfalls eine Vielzahl von taktischen Aufgaben für das Informationsmanagement. Es entstehen personenbezogene Daten, für deren Umgang hohe Anforderungen an den Datenschutz existieren. Diese Anforderungen müssen analysiert werden, so dass ein Konzept erstellt werden kann, das sowohl den Datenschutz berücksichtigt, als auch die Interessen zur Nutzung der Daten einbezieht. Hier gibt es erste technische Ansätze zur Lösung der Problematik, aber keine übergeordnete $\mathrm{Be}$ trachtung aus der Sicht des Informationsmanagements.

Bei der Datengenerierung wäre es sinnvoll, herstellerübergreifende Standards zu entwickeln, damit Stromzähler nicht bei etwaigen Anbieterwechseln ausgetauscht werden müssten. Zur Datenübertragung existieren verschiedene Technologien, die im Hinblick auf Sicherheit, Zuverlässigkeit und Kosteneffizienz analysiert werden müssten.

Weiterhin ist nicht klar, in welcher Granularität die Daten zum Stromverbrauch, Netzauslastung, Frequenzhaltung übertragen und gespeichert werden müssten, um die nötigen Informationen zum Nutzerverhalten und zur Netzsteuerung zu erhalten, ohne die entstehende Datenmenge unnötig zu vergrößern.
Die operativen Aufgaben bestehen u. a. darin, zu gewährleisten, dass ein komplexes, IT-gesteuertes Stromnetz gegen Angriffe von außen geschützt ist. Interne Ausfälle müssen so abgefangen werden können, dass die Versorgungssicherheit nicht gefährdet ist.

Das entstehende Volumen der Daten ist bei ca. 40,2 Mio. Haushalten in Deutschland (Statistisches Bundesamt 2011) und z. B. stündlicher oder sogar minütlicher Übermittlung gewaltig. Die Verarbeitung dieser ständig wiederholt anfallenden Massendaten stellt eine große Herausforderung für das Informationsmanagement z. B. für die Architektur von Standardanwendungssoftware dar.

\section{Zusammenfassung und Ausblick}

Die grundsätzliche Notwendigkeit des Smart Metering in einem durch die Einspeisung erneuerbarer Energien volatiler und dezentraler werdenden Energieversorgungssystem sind erkannt, dennoch befindet sich die Umsetzung noch in den Anfängen.

Investitionen müssen refinanziert werden, am wirtschaftlichen Nutzen wollen unterschiedliche Marktteilnehmer mit z. T. partikularen und konfliktären Interessen partizipieren (u. a. Energieerzeuger, Netzbetreiber, Versorger und Verteiler, Messstellenbetreiber und Dienstleister). Nicht zuletzt erwarten die Kunden deutliche Mehrwerte durch entsprechende Serviceangebote mit flexiblen Tarifen.

Wachsender regulatorischer Druck zu mehr Energieeffizienz, steigendes Nachhaltigkeitsbewusstsein der Nutzer, der zunehmende Anteil der dezentralen Energieerzeugung, innovative Serviceangebote und neue Anwendungen für die Nutzer werden die Einführung der Smart-Metering-Technologie fördern.

Art und Umfang der dargestellten Herausforderungen belegen, dass das Informationsmanagement und die Wirtschaftsinformatik insgesamt einen Beitrag zur Lösung der Aufgaben, die aus dem Einsatz des Smart Metering resultieren, leisten kann und muss.

Open Access Dieser Artikel unterliegt den Bedingungen der Creative Commons Attribution Noncommercial License. Dadurch sind die nichtkommerzielle Nutzung, Verteilung und Reproduktion erlaubt, sofern der/die Originalautor/en und die Quelle angegeben sind.

\section{Literatur}

BMU und BMWi (2010) Energiekonzept Neun Punkte für eine umweltschonende, zuverlässige und bezahlbare Energieversorgung. http://www.erneuerbareenergien.de/inhalt/46394/4590/. Abruf am 2011-01-04

BMWi (2011a) Intelligente Netze und intelligente Zähler - Smart Grids/Smart Meter. http://www.bmwi.de/BMWi/Navigation/ Energie/Energietraeger/netze,did=354346. html. Abruf am 2011-01-06

BMWi (2011b) Smart Watts - Leuchtturmprojekt E-Energy. http://www.smartwatts.de/ das-internet-der-energie/

leuchtturmprojekt-e-energy.html. Abruf am 2011-04-01

EnWG (2005) Gesetz über die Elektrizitätsund Gasversorgung. http://www.gesetzeim-internet.de/enwg_2005/index.html. Abruf am 2011-04-05

ERGEG (2007) Smart metering with a focus on electricity regulation. http://www. energy-regulators.eu/portal/page/portal/ EER HOME/EER PUBLICATIONS/CEER ERGEG_PAPERS/Customers/2007/E07- 
RMF-04-03 SmartMetering 2007-10-31 0. pdf. Abruf am 2011-04-05

ESMA (2010) What is smart metering? European smart metering alliance. http:// www.esma-home.eu/smartMetering/. Abruf am 2010-11-25

EU-Kommission (2007) 20 und 20 bis 2020 Chancen Europas im Klimawandel. http:// eur-lex.europa.eu/LexUriServ/LexUriServ. do?uri=COM:2008:0030:FIN:de:PDF. Abruf am 2011-04-05

Europäische Union (2006) Richtlinie 2006/32/EG des Europäischen Parlaments und des Rates über Endenergieeffizienz und Energiedienstleistungen und zur Aufhebung der Richtlinie 93/76/ EWG des Rates. http://eur-lex.europa.eu/ LexUriServ/LexUriServ.do?uri=OJ:L:2006: 114:0064:0064:DE:PDF. Abruf am 2011-0405
Fox-Penner P (2010) Smart power: climate change, the smart grid, and the future of electric utilities. Island Press, Washington

Heinrich LJ, Lehner F (2005) Informationsmanagement: Planung, Überwachung und Steuerung der Informationsinfrastruktur. Oldenbourg Wissenschaftsverlag München

Müller-Elschner C (2010) Die Rolle von Informations- und Kommunikationstechnologie beim Smart Metering; Smarte Netze für smarte Nutzer. In: Köhler-Schute C (Hrsg) Smart Metering: Technologische, wirtschaftliche und juristische Aspekte des Smart Metering. Ks-Energy-Verlag, Berlin

Österle H, Winter R (2003) Business Engineering: Auf dem Weg zum Unternehmen des Informationszeitalters. Springer, Berlin

Statistisches Bundesamt (2011) Demografischer Wandel in Deutschland - Heft 1 -
Bevölkerungs- und Haushaltsentwicklung im Bund und in den Ländern. http://www. destatis.de/jetspeed/portal/cms/Sites/ destatis/Internet/DE/Content/ Publikationen/Fachveroeffentlichungen/ Bevoelkerung/

VorausberechnungBevoelkerung/ BevoelkerungsHaushaltsentwicklung 5871101119004,property=file.pdf. Abruf am 2011-04-05

VDE/ITG (2010) Energieinformationsnetze und -systeme: Bestandsaufnahme und Entwicklungstendenzen. Ein Positionspapier der Informationstechnischen Gesellschaft im VDE (ITG)

Watson R, Boudreau M, Chen A (2010) Information systems and environmentally sustainable development: energy informatics and new directions for the IS community. Management Information Systems Quarterly 34(1):23-38 